\title{
Neurofilaments Consist of Distinct Populations That Can Be Distinguished by C-Terminal Phosphorylation, Bundling, and Axonal Transport Rate in Growing Axonal Neurites
}

\author{
Jason T. Yabe, ${ }^{1}$ Teresa Chylinski, ${ }^{1}$ Feng-Song Wang, ${ }^{2}$ Aurea Pimenta, ${ }^{3}$ Solomon D. Kattar, ${ }^{1}$ \\ Maria-Dawn Linsley, ${ }^{1}$ Walter K-H Chan, ${ }^{1}$ and Thomas B. Shea ${ }^{1}$ \\ ${ }^{1}$ Center for Cellular Neurobiology and Neurodegeneration Research, Department of Biological Sciences, University of \\ Massachusetts-Lowell, Lowell, Massachusetts 01854, 2Department of Biological Sciences, Purdue University-Calumet, \\ Hammond, Indiana 46323, and '3Department of Neurobiology, University of Pittsburgh School of Medicine, Pittsburgh, PA 15261
}

We examined the steady-state distribution and axonal transport of neurofilament (NF) subunits within growing axonal neurites of $\mathrm{NB} 2 \mathrm{a} / \mathrm{d} 1$ cells. Ultrastructural analyses demonstrated a longitudinally oriented "bundle" of closely apposed NFs that was surrounded by more widely spaced individual NFs. NF bundles were recovered during fractionation and could be isolated from individual NFs by sedimentation through sucrose. Immunoreactivity toward the restrictive C-terminal phospho-dependent antibody RT97 was significantly more prominent on bundled than on individual NFs. Microinjected biotinylated NF subunits, GFP-tagged NF subunits expressed after transfection, and radiolabeled endogenous subunits all associated with individual NFs before they associated with bundled NFs. Biotinylated and GFP-tagged NF subunits did not accumulate uniformly along bundled NFs; they initially appeared within the proximal portion of the NF bundle and only subsequently were observed along

Despite decades of study, controversy exists over several aspects of neurofilament (NF) axonal transport. Models that have been advanced to describe the organization of axonal NFs range from relentless translocation of a homogeneous population of NFs (Lasek, 1986; Lasek et al., 1992, 1993) to translocation of only some NFs, whereas others incorporate into a distinct cytoskeletal macrostructure that is essentially stationary (Nixon and Logvinenko, 1986; Nixon, 1993, 1998). Contemporary articulation of these models demonstrates them to display considerable inherent similarity in that they both consider that NFs transport over a broad range of rates and that these rates are derived from the relative length of time the individual NFs are associated with their transport vector (Lasek et al., 1993; Nixon, 1993, 1998). The essential difference among these models is whether axonal NFs constitute a single population with a continuum of transport rates or whether the differences in transport rates actually characterize distinct NF populations.

Received Oct. 19, 2000; revised Dec. 12, 2000; accepted Dec. 15, 2000.

This research was supported by the National Science Foundation and the National Institute of Child Health and Human Development. We thank our colleagues Anthony Brown and Ralph Nixon for their helpful comments on this manuscript, Ron Liem for his generous gift of neurofilament cDNA, Daniela Ortiz for technical assistance, and Louise Trakimas for excellent assistance with electron microscopy.

Correspondence should be addressed to Dr. Thomas B. Shea, Center for Cellular Neurobiology and Neurodegeneration Research, Department of Biological Sciences, University of Massachusetts-Lowell, One University Avenue, Lowell, MA 01854. E-mail: Thomas_Shea@uml.edu.

Copyright () 2001 Society for Neuroscience 0270-6474/01/212195-11\$15.00/0 the entire length of bundled NFs. These findings demonstrate that axonal NFs are not homogeneous but, rather, consist of distinct populations. One of these is characterized by less extensive C-terminal phosphorylation and a relative lack of NF-NF interactions. The other is characterized by more extensive C-terminal NF phosphorylation and increased NF-NF interactions and either undergoes markedly slower axonal transport or does not transport and undergoes turnover via subunit and/or filament exchange with individual NFs. Inhibition of phosphatase activities increased NF-NF interactions within living cells. These findings collectively suggest that C-terminal phosphorylation and NF-NF interactions are responsible for slowing NF axonal transport.

Key words: neurofilaments; axonal transport; cytoskeleton; axon; phosphorylation; neuronal differentiation

The form in which NF subunits undergo transport is also controversial. Various studies provide evidence for the transport of NFs themselves (Nixon and Lewis, 1986; Galbraith et al., 1999; Koehnle and Brown, 1999; Wang et al., 2000), transport of nonfilamentous punctate assemblies of NF subunits (Yabe et al., 1999), and transport of monomers or small oligomers (Takeda et al., 1994; Terada et al., 1994; Hirokawa et al., 1997). Studies demonstrating the transport of nonfilamentous punctate assemblies demonstrate that they can assume filamentous form during transport and consider punctate structures only as transitional forms (Yabe et al., 1999). Such studies, and those indicating transport of filaments, are consistent with the possibility that axonal NFs undergo turnover chiefly via replacement with new NF polymer (for review, see Baas and Brown, 1997). Conversely, studies supporting transport of monomers and/or small oligomers are interpreted to indicate that the bulk of axonal NFs does not itself translocate and instead undergoes turnover by exchange/ incorporation of transporting subunits (for review, see Hirokawa et al., 1997).

Finally, controversy also exists regarding the nature of NF interactions within axons and regarding how NFs exert their stabilizing influences on axonal cytoarchitecture. Certain studies indicate that NFs undergo phosphorylation-dependent crosslinking and bundling (Leterrier and Eyer, 1987; Shaw and Hou, 1990; Nagakawa et al., 1995; Leterrier et al., 1996). Resultant formation of an interconnected suprastructure could readily explain how NFs impart support to the axon. However, the dem- 
onstration that NFs move apart on disruption of axonal membrane integrity suggests that at least the majority of NFs is not cross-linked physically (Brown and Lasek, 1993). NFs still can be envisioned to impart stability to axons in the absence of physical cross-linking with the consideration that their extensive phosphorylation fosters $\mathrm{NF}-\mathrm{NF}$ repulsion and restricts their lateral motion.

In the present study we addressed the above controversies by examining the organization and dynamics of NFs in growing axonal neurites in culture.

\section{MATERIALS AND METHODS}

Cell culture. NB2a/d1 cells were selected for these analyses because (1) they express and phosphorylate all three NF subunits (Shea et al., 1988, 1990), and (2) they accumulate a prominent bundle of Triton-insoluble axonal NFs that resembles NF bundles observed in axons in situ (Shaw and Hou, 1990). In addition, although some "bundled" axonal NFs also have been reported in certain cultured neurons (Brown, 1997), the ability to generate large quantities of NB2a/d1 cells and to control their differentiation state renders them particularly suitable for biochemical as well as immunological and ultrastructural analyses.

Cells were cultured on poly-L-lysine-coated plates or coverslips in DMEM (high glucose formulation) containing 10\% horse serum. Elaboration of axonal neurites was induced by the addition of $1 \mathrm{~mm}$ dibutyryl cyclic AMP (dbcAMP) for $3 \mathrm{~d}$ (Shea et al., 1988). In some experiments the cells received $5 \mathrm{~nm}$ okadaic acid (OA) for the final $24 \mathrm{hr}$ of differentiation (Shea et al., 1993). In some experiments at either 2 or $18 \mathrm{hr}$ after microinjection, some cells were treated with $330 \mathrm{~nm}$ nocodazole for $2 \mathrm{hr}$. Resultant perturbation of axonal microtubules by this treatment induces overall thinning of axonal caliber with periodic "beading" (Shea and Beermann, 1994).

Biotinylation and microinjection of NF subunits. Bovine spinal cords were homogenized in $100 \mathrm{~mm}$ PIPES, $\mathrm{pH}$ 6.6, containing (in $\mathrm{mM}$ ) 1 EGTA, $1 \mathrm{MgCl}_{2}, 1 \mathrm{PMSF}$, and 1 DTT plus $1 \mu \mathrm{g} / \mathrm{ml}$ leupeptin. NFs were recovered in the void volume after Sepharose CL-4B gel chromatography (Takeda et al., 1994). As described (Takeda et al., 1994), a single assembly/disassembly cycle was sufficient to eliminate tubulin and other contaminating proteins. Isolated NFs were disassembled in urea buffer [100 mM phosphate buffer, $\mathrm{pH} 7.5$, containing $6 \mathrm{M}$ urea, $0.5 \mathrm{M}$ PMSF, 1 $\mu \mathrm{g} / \mathrm{ml}$ leupeptin, and (in mM) 1 EDTA, 1 EGTA, and 1 DTT] and then dialyzed overnight at $4^{\circ} \mathrm{C}$, followed by $1 \mathrm{hr}$ at $37^{\circ} \mathrm{C}$ in assembly buffer [containing (in mM) 20 PIPES, pH 6.6, 1 EGTA, $1 \mathrm{MgCl}_{2}, 1$ EDTA, 1 DTT, $0.17 \mathrm{NaCl}$ (Takeda et al., 1994)]; they were recovered by centrifugation at $100,000 \times g$ for $1 \mathrm{hr}$. The resultant once-cycled NFs were conjugated with 3-( $N$-maleimidylproprionyl) biocytin $(25 \mathrm{ng} / \mathrm{ml}$; Molecular Probes, Eugene, OR) while in assembled form essentially as described previously (Takeda et al., 1994). NF-H and NF-L subsequently were purified by DE-52 chromatography in urea buffer; pure fractions (ascertained by SDS-gel electrophoresis and immunoblot analysis; see Results) were combined and dialyzed overnight at $4^{\circ} \mathrm{C}$ against assembly buffer to remove urea and then dialyzed for $6 \mathrm{hr}$ against 10,000 volumes of injection buffer [containing (in mM) 5 HEPES, 1 DTT, 1 EDTA, and 1 EGTA, pH 8.5], concentrated by centrifugation onto Centricon filters, and stored at $-80^{\circ} \mathrm{C}$ (Takeda et al., 1994). Just before injection, purified subunits were mixed 1:1 with $70 \mathrm{kDa}$ fluorescein-conjugated dextran (Molecular Probes) and clarified by centrifugation for $10 \mathrm{~min}$ in a Beckman airfuge; the resulting supernatant (containing $\sim 1 \mathrm{mg} / \mathrm{ml} \mathrm{NF}$ protein) was microinjected as described (Straube-West et al., 1996; Jung et al., 1998; Yabe et al., 1999). Injected cells were located under fluorescein optics and examined by phase-contrast microscopy; cells exhibiting any obvious trauma resulting from microinjection were excluded from further analyses. Notably, translocation of microinjected subunits into axonal neurites of NB2a/d1 cells was prevented by administration of the anti-microtubule drug nocodazole immediately after microinjection (Jung et al., 1998; Yabe et al., 1999), indicating that microinjected subunits required an intact microtubule network to undergo active transport. These data confirm that no significant contribution to the transport of biotinylated subunits into neurites is derived from subunit diffusion and/or injection pressure.

Immunofluorescence and immunoelectron microscopy. Cells were processed for endogenous and microinjected NF immunoreactivity at 2-18 $\mathrm{hr}$ after microinjection. To preserve axonal microtubules (MTs), we rinsed the cells in warm Tris-buffered saline, pH 7.4 (TBS), and fixed them under MT-stabilizing conditions [containing (in mM) 60 PIPES, $\mathrm{pH}$ 6.9, $10 \mathrm{EGTA}$, and $2 \mathrm{MgCl}_{2}$ plus $10 \mu \mathrm{M}$ taxol (Shea, 1999)]; alternate cultures also received $1 \%$ saponin to extract unassembled tubulin subunits. Alternatively, to deplete MTs and associated proteins (some of which may cross-react with phospho-dependent anti-NF antibodies), we fixed most cells at $4^{\circ} \mathrm{C}$ in the absence of taxol in $50 \mathrm{~mm}$ Tris, $\mathrm{pH} 6.8$, containing $5 \mathrm{~mm}$ EDTA, $1 \mathrm{~mm}$ PMSF, and $50 \mu \mathrm{g} / \mathrm{ml}$ leupeptin, with and without previous extraction for $15 \mathrm{~min}$ at $4^{\circ} \mathrm{C}$ with $1 \%$ Triton $\mathrm{X}-100$ (Shea et al., 1988). Triton extraction under these conditions also depletes some NFs (Shea et al., 1990). Nonextracted cells were incubated at $4^{\circ} \mathrm{C}$ in the same buffer without Triton X-100; then all cultures were fixed for 15 min with $4 \%$ paraformaldehyde in TBS and permeabilized by incubation with $0.1 \%$ Triton $\mathrm{X}-100$ for $15 \mathrm{~min}$ at room temperature. Because fixation without taxol at $4^{\circ} \mathrm{C}$ effectively depleted most MTs and more clearly visualized the NFs (see Fig. $1 A-C$ ), all cells except those in Figure $1, A$ and $B$, were subjected to this latter Triton X-100 extraction and fixation protocol. Some additional cultures were processed by using a "splaying" technique (Brown, 1998) that affords visualization of filamentous profiles.

Cultures were immunostained with 1:100 dilutions of monoclonal antibodies directed toward phosphorylated (SMI-31) and nonphosphorylated (SMI-32) NF epitopes (Sternberger Monoclonals, Bethesda, MD), a monoclonal antibody (RT97) directed against a developmentally delayed phospho-epitope of NF-H (Anderton et al., 1982), a polyclonal antibody generated in this laboratory against electrophoretically purified murine NF-L (L3), or a polyclonal anti-biotin antibody (Jung et al., 1998), followed by 1:150 dilutions of rhodamine- or fluoresceinconjugated anti-mouse or anti-rabbit IgG. Incubations with primary antibodies were done either overnight at $4^{\circ} \mathrm{C}$ or for $2 \mathrm{hr}$ at $37^{\circ} \mathrm{C}$. Incubations with secondary antibodies were for $2 \mathrm{hr}$ at $37^{\circ} \mathrm{C}$. For doubleimmunofluorescent analyses the primary antibodies were applied simultaneously and, after rinsing, secondary antibodies also were applied simultaneously.

For immunoelectron microscopy (EM), cells extracted with $1 \%$ Triton $\mathrm{X}-100$ at $4^{\circ} \mathrm{C}$ (see above) were fixed in $4 \%$ glutaraldehyde at $4^{\circ} \mathrm{C}$; rinsed; incubated (1 hr at room temperature) with 1:100 dilutions of SMI-31, RT97, or anti-biotin; rinsed three times; and then incubated with secondary antibodies conjugated to 5 or $10 \mathrm{~nm}$ colloidal gold particles. Samples were dehydrated, embedded in resin, sectioned, and stained with uranyl acetate by conventional methods and examined in a Philips 300 EM (Shea et al., 1993). Positive identification of injected cells required embedding within the culture dish and sectioning parallel to the substrate; because of the difficulty of obtaining the perikaryon and the entire axonal length $(100 \mu \mathrm{m}$; Shea, 1999) within individual sections, many analyses were performed on proximal or distal regions of individual injected cells.

Construction of eGFP-NF-M and transfection. As described previously (Yabe et al., 1999), a 2484 bp fragment encoding amino acids 1-824 of rat NF-M cDNA was isolated from the plasmid pRSVi-NF-M (generous gift of Dr. Ron Liem, Columbia University, New York, NY; Chin and Liem, 1989) by a double-restriction enzyme digestion with HindIII and HincII (blunt end), followed by agarose gel electrophoresis and gel extraction with JetSorb (Genomed, Raleigh, NC). The purified fragment was subcloned into the HindIII and SmaI (blunt end) restriction sites of the eukaryotic expression vector peGFP-N3 (Clontech, Palo Alto, CA) in frame with eGFP. After overnight ligation at $12^{\circ} \mathrm{C}$ the reaction mixture was used to transform HB101 Escherichia coli. Ampicillin-resistant colonies were isolated for mini-preparations of plasmid, which were analyzed by restriction digestion for the NF-M cDNA fused in frame with eGFP. Large-scale plasmid preparations for transfections were performed with the Qiagen purification system (Qiagen, Santa Clarita, CA) to generate in transfected cell lines NF-M as a fusion protein with eGFP. All standard molecular biology procedures were performed essentially as described by Sambrook et al. (1989).

NB2a/d1 cells treated with dbcAMP for $2 \mathrm{~d}$ were transfected with 33.2 $\mu \mathrm{g} / \mathrm{ml}$ eGFP-NF-M or eGFP without an NF-M insert in $238 \mu \mathrm{g} / \mathrm{ml}$ Superfect (Qiagen) for $3 \mathrm{hr}$ in the presence of $10 \%$ serum, after which the medium was replaced (Yabe et al., 1999). Cells were incubated for an additional $24 \mathrm{hr}$ to allow for accumulation of eGFP-NF-M and for continued elaboration of axonal neurites Stably transfected cells were selected by the addition of $100 \mu \mathrm{g} / \mathrm{ml}$ GD14 (Life Technologies, Grand Island, NY) to the medium and the isolation of surviving colonies 7-14 d later. Accumulation of full-length eGFP-conjugated NF-M was confirmed by immunoblot analyses of stably transfected cultures (see below). Densitometric analyses. Phase-contrast and epifluorescent images of 
individual cells were captured via a Dage CCL-72 camera connected to a Scion LG-3 frame grabber housed in a Macintosh 7100AV and operated by NIH Image software. Images were stored as TIFF or PICT files.

The relative distribution of NF immunoreactivity was determined via NIH Image software. Only cells elaborating a single unbranching axonal neurite were included in analyses. After automated background subtraction, areas of axonal neurites were encircled by using the freehand tool of the program, and the resultant net densitometric value was recorded. For determination of the relative distribution of NF immunoreactivity within nocodazole-induced beads and thinned areas of the shaft, representative beads were encircled, and the resulting densitometric value was recorded. Then the identical circle was shifted to an adjacent nonbeaded area of the axonal shaft. The ratio of immunoreactivity within beads versus adjacent areas of the axonal shaft was obtained by dividing the densitometric value of a given bead by that of its adjacent shaft. Values that are presented represent the mean and SEM derived from six to eight injected cells from two separate experiments. Statistical comparisons were performed via Student's $t$ test.

To generate mean distribution profiles for multiple axons of transfected cells, we measured the contour length of axonal neurites in the digitized phase-contrast image by using the "neurite labeling macro" of the NIH program. The resulting length was divided into 10 equivalent segments. The start of segment one in these analyses was defined as that region of the axon shaft that was distinguished clearly from the perikaryon and any gradual thinning of the perikaryon. Accordingly, this first segment was entirely within the axonal shaft and did not include the axonal hillock. Similarly, the end of segment 10 was defined as the final segment of the shaft just before the swelling of the growth cone. Then the density of each segment in the corresponding epifluorescent image was calculated by drawing a box around each segment, shifting the box to an immediately adjacent cell-free area, and recording individual background densities. The box was shifted as little as possible for background recording, which maintained the relative spatial localization within the microscopic field of the selected background area relevant to the corresponding selected axonal area; this eliminated the potential influence of differential illumination across a given microscopic field. Resultant data were exported to Excel spreadsheet software for subsequent calculations.

In additional analyses the relative distribution of colloidal gold particles in immuno-EM analyses was quantified within proximal or distal regions of axonal neurites of differentiated NB2a/d1 cells or within bundled NFs versus adjacent regions of the same axons not containing bundled NFs. In such analyses the percentage of gold particles within the region of interest was calculated relative to the total number of particles within the region of interest plus the adjacent/opposing region.

Gel electrophoresis, autoradiography, and immunoblot analyses. dbcAMP-treated cells on uncoated plates were pulse-radiolabeled for 15 min with $\left[{ }^{35}\right.$ S $]$ methionine in methionine-free DMEM without serum $\left(500 \mu \mathrm{C}\right.$ i for each of $2 \times 10 \mathrm{~cm}^{2}$ plates at $\sim 80 \%$ confluency; Shea et al., 1990). Some cultures were harvested immediately after labeling. For additional cultures the medium was replaced with medium lacking radiolabel but containing $10 \%$ horse serum and $20 \times$ the normal concentration of methionine; incubation was continued for a total of $4 \mathrm{hr}$ before harvesting. All cultures were incubated in methionine-free, serum-free medium for $15 \mathrm{~min}$ before radiolabeling.

Radiolabeled cells were harvested according to established methods that separate axonal neurites from perikarya (Shea et al., 1993). Briefly, cultures were harvested by scraping with a rubber policeman in $50 \mathrm{~mm}$ Tris-HCl, pH 6.8, containing 5 mM EDTA, $1 \mathrm{~mm} \mathrm{PMSF}$, and $50 \mu \mathrm{g} / \mathrm{ml}$ leupeptin; they were homogenized gently on ice ( 25 strokes) in a loosefitting glass-Teflon homogenizer and then centrifuged at $5000 \times g$ for 5 $\min$ at $4^{\circ} \mathrm{C}$. This procedure sediments axonal fragments but leaves disrupted perikarya in the supernatant (Shea et al., 1993). The axonal pellet was resuspended in the same buffer containing $1 \%$ Triton X-100, and the perikaryal supernatant was made $1 \%$ Triton X-100. Cytoskeletons then were isolated separately from axonal- and perikaryal-enriched fractions by homogenization (50 strokes in a tight-fitting glass-Teflon homogenizer) on ice, followed by centrifugation at $15,000 \times g$ for $15 \mathrm{~min}$ at $4^{\circ} \mathrm{C}$ (Shea et al., 1988). Axonal cytoskeletons were resuspended in the same buffer without Triton and then sedimented for $15 \mathrm{~min}$ at $15,000 \times$ $g$ onto $1 \mathrm{ml}$ of $1 \mathrm{M}$ sucrose in the same buffer. Aliquots of cytoskeletons from cell bodies and from the interface and pellet generated after the above sucrose gradient centrif ugation were immunoprecipitated with a 1:150 dilution of a polyclonal antibody (R39) that precipitates all NF subunits (Shea et al., 1997; Jung et al., 1998). Gels were dried, and autoradiographs were generated. Aliquots of immunoprecipitated mate- rial also were subjected to immunoblot analyses with R39. Autoradiographs and immunoblots were digitized with a UMAX flatbed scanner equipped with a "transparency adaptor" at 300 dpi (Jung and Shea, 1999).

Immunoblots of Triton-insoluble cytoskeletons $(100 \mu \mathrm{g})$ from stably transfected cells were probed with a polyclonal anti-GFP antibody (Clontech) and a polyclonal antibody (M2) generated in this laboratory against NF-M.

\section{RESULTS}

\section{Axonal neurites contain a core of closely opposed NFs that exhibit increased site-specific C-terminal phosphorylation and increased NF-NF interactions}

Consistent with previous studies (Shea et al., 1988), intermediatesized filaments were detected along the length of axonal neurites (Fig. 1A-C). Reactivity with the relatively nonrestrictive (Shea et al., 1989) phospho-dependent antibody SMI-31 indicated that these intermediate filaments were NFs (Fig. 1C,D). Depletion of axonal MTs highlighted that a significant portion of Tritoninsoluble axonal NFs was concentrated within the center of the axon with respect to the longitudinal axis (Fig. $1 C-E$ ); these closely apposed NFs will be referred to as "bundles" for the remainder of this report. Bundled NFs were situated at a mean distance of $\sim 17.5 \pm 1.7 \mathrm{~nm}$ apart. The closest peripherally situated individual NFs averaged $34.3 \pm 5.3 \mathrm{~nm}$ apart and ranged to $>60 \mathrm{~nm}$ apart. Previous analyses (Gotow and Tanaka, 1994) have demonstrated that bundled NFs could be distinguished from individual axonal NFs by the increased prevalence of a developmentally delayed phospho-epitope (RT97; Anderton et al., 1982). We therefore examined whether this was also the case in NB2a/d1 axonal neurites. Consistent with this possibility, SMI-31 immunoreactivity was distributed relatively evenly throughout the axonal width, whereas RT97 was relatively more concentrated within the central aspect of axonal neurites (Fig. $1 D-F)$. Immuno-EM analyses confirmed that bundled NFs displayed more than fourfold increased affinity $(p<0.01)$ for RT97 than did individual NFs (Fig. $1 E$ ), whereas individual and bundled NFs displayed equivalent SMI-31 immunoreactivity. Restriction of RT97 immunoreactivity within the central bundle was highlighted further, comparing the distribution of NF-L (as an index of total NFs) with that of RT97 or SMI-31; SMI-31 codistributed throughout the axonal diameter with NF-L, whereas RT97 was concentrated along the center of axonal neurites (Fig. $1 F$ ).

Also consistent with previous studies (Shaw and Hou, 1990; Leterrier et al., 1996), bundled NFs were recovered among individual NFs in cytoskeletal preparations derived from NB2a/d1 axonal neurites; bundles observed within such preparations also demonstrated markedly more RT97 immunoreactivity than did individual NFs (Fig. 2). The bulk of axonal NFs moves apart when freed from the constraints of the axonal membrane, suggesting that they are not cross-linked physically within axons (Brown and Lasek, 1993). However, recovery of some bundled NFs previously has been interpreted to indicate that a population of NFs indeed is cross-linked in some capacity in situ (Shaw and Hou, 1990). Bundled NFs within NB2a/d1 axonal neurites therefore may represent a population of NFs that have undergone more extensive NF-NF interactions than the more peripherally situated individual NFs. In support of this notion the NF bundles, but not individual NFs, sedimented through $1 \mathrm{M}$ sucrose (Fig. 2).

Because NF phosphorylation has been reported to mediate NF bundling in cell-free analyses, we undertook to determine whether we could modulate the extent of NF bundling within axonal neurites. To accomplish this, we added the phosphatase inhibitor okadaic acid (OA) to cultures for the final $24 \mathrm{hr}$ of their 
Figure 1. NB2a/d1 axonal neurites contain a mixture of closely opposed and relatively disbursed NFs that exhibit differential reactivity toward phosphodependent NF antibodies. $A-C$, Regions of axonal neurites of differentiated NB2a/d1 cells. $A, B$, Neurites fixed at $37^{\circ} \mathrm{C}$ under MT-stabilizing conditions in the absence $(A)$ and presence $(B)$ of $1 \%$ saponin (Shea, 1999). Note prominent MT profiles along the neurite. $C$, An axonal neurite extracted with $1 \%$ Triton X-100 at $4^{\circ} \mathrm{C}$ in the absence of MT-stabilizing agents. Note the centrally located "bundle" of closely apposed NFs (arrows) and the relative depletion of MTs. On the basis of these findings, all cultures for the remainder of this study were fixed at $4^{\circ} \mathrm{C}$, with or without Triton X-100 and in the absence of taxol, to deplete axonal MTs and to reveal more clearly the Triton-insoluble NFs. $A-C$ are presented at the same magnification. $D$, Axonal neurites of cells processed for immuno-EM with SMI-31 or RT97, as indicated, followed by colloidal gold-conjugated secondary antibody. $C$ presents an axonal neurite, at smaller magnification, which also was processed for immuno-EM with SMI-31. Note that SMI-31 immunoreactivity is distributed among bundled and nonbundled individual NFs, whereas RT97 immunoreactivity is localized to bundled NFs. Arrows in the neurite that was reacted with SMI-31 denote individual NFs (peripheral to the bundle) that are decorated with colloidal gold, whereas arrows in the neurite reacted with RT97 denote individual NFs that are not decorated with colloidal gold. E, Quantification of the distribution of SMI-31 and RT97 immunoreactivity on bundled and peripherally located individual NFs. Note that the SMI-31 epitope is distributed relatively evenly between bundled and individual NFs, whereas the RT97 epitope is markedly more prevalent on bundled NFs. $F$, Double-immunofluorescent analyses of the distribution of SMI-31 and NF-L and, in a second cell, RT97 and NF-L. NF-L immunoreactivity is distributed evenly throughout axonal neurites. Note that, like NF-L immunoreactivity, SMI-31 immunoreactivity is distributed relatively evenly throughout axonal neurites. RT97 immunoreactivity, by contrast, is relatively concentrated along the center of the axon with respect to its longitudinal axis and does not codistribute with NF-L.
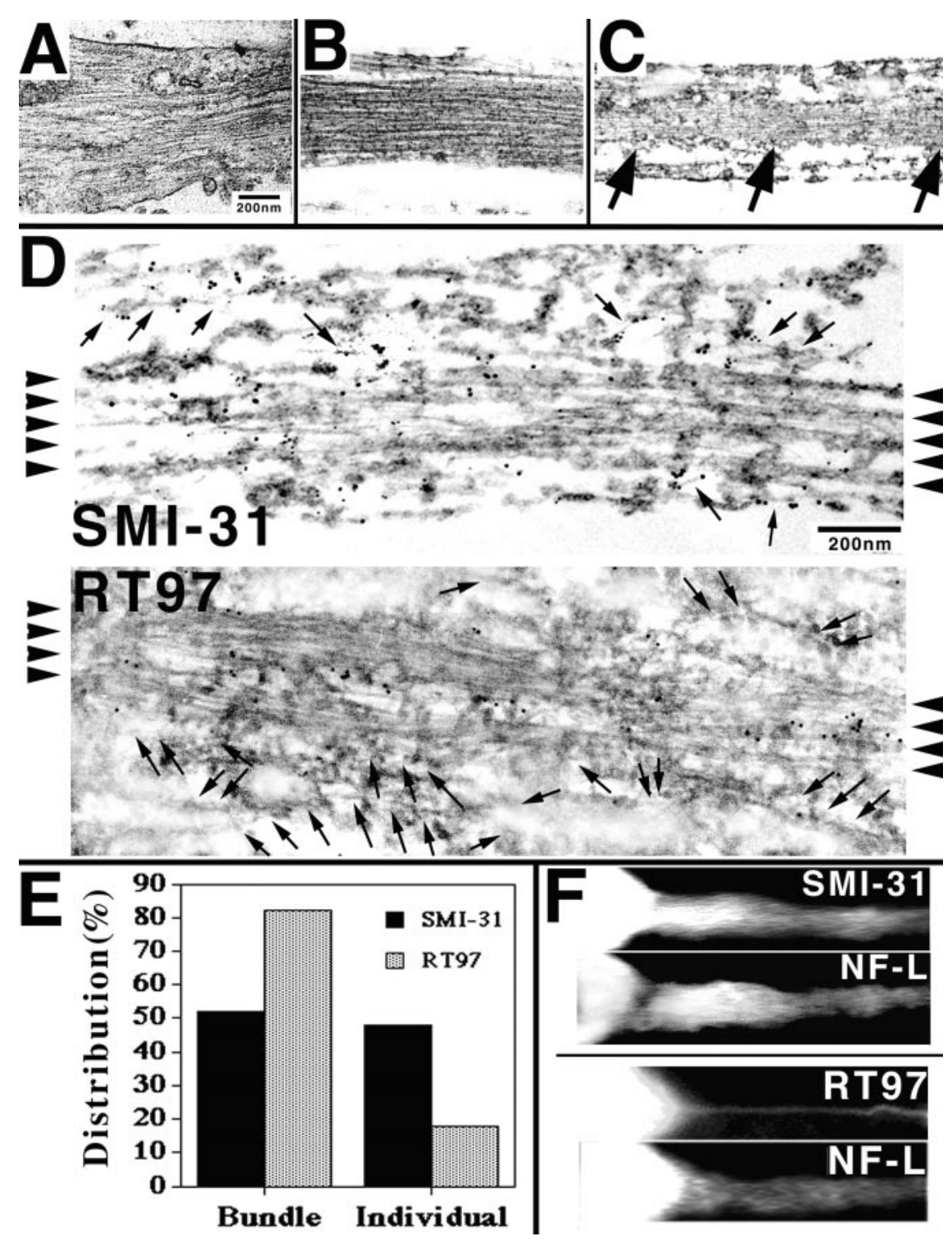

dbcAMP treatment. OA previously has been reported to increase the number of phospho-NFs within these cells (Shea et al., 1993), to mediate increased C-terminal NF phosphorylation, and to decrease NF axonal transport rate in situ (Veeranna et al., 1995; Jung and Shea, 1999). We then quantified the number of SMIserved within $20 \mathrm{~nm}$ of each other along their longitudinal length in longitudinally oriented thin sections of axonal neurites from multiple OA-treated and untreated cells; this distance was selected on the basis of mean NF-NF distance within centrally 31-reactive filaments (as an index of total NFs) that were ob-

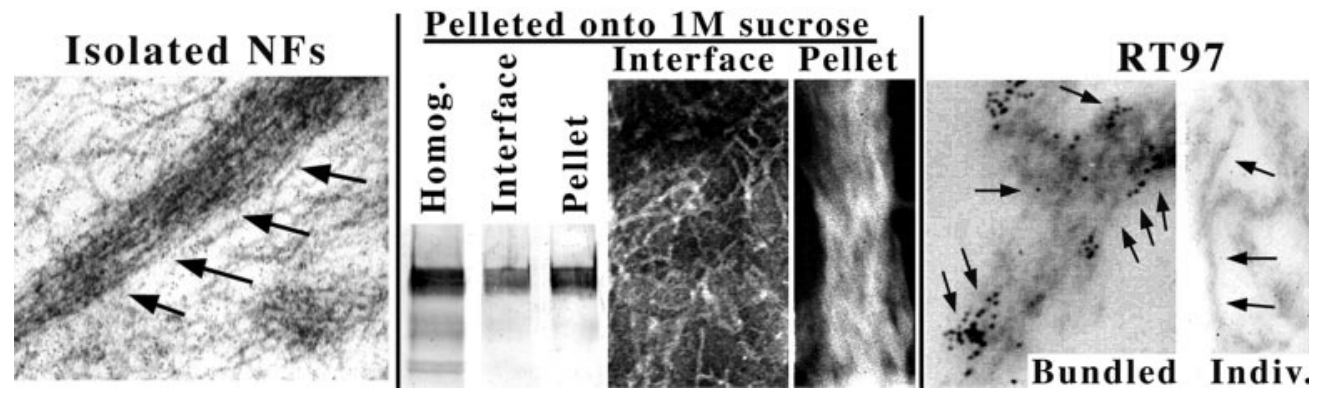

Figure 2. Some NFs are recovered from axonal neurites in bundled form. The panels present ultrastructural and immunological analyses of cytoskeletons. Bundled NFs were detected among individual NFs in sectioned cytoskeletal preparation (Isolated NFs). Negative stain analyses demonstrated that bundles sedimented via $1 \mathrm{M}$ sucrose, whereas individual NFs did not (Pelleted onto 1 M sucrose); the accompanying immunoblots were processed for SMI-31 immunoreactivity, and the $200 \mathrm{kDa}$ region is presented. Immuno-EM analyses demonstrated that RT97 immunoreactivity was associated selectively with bundled NFs in sectioned cytoskeletal preparations. 

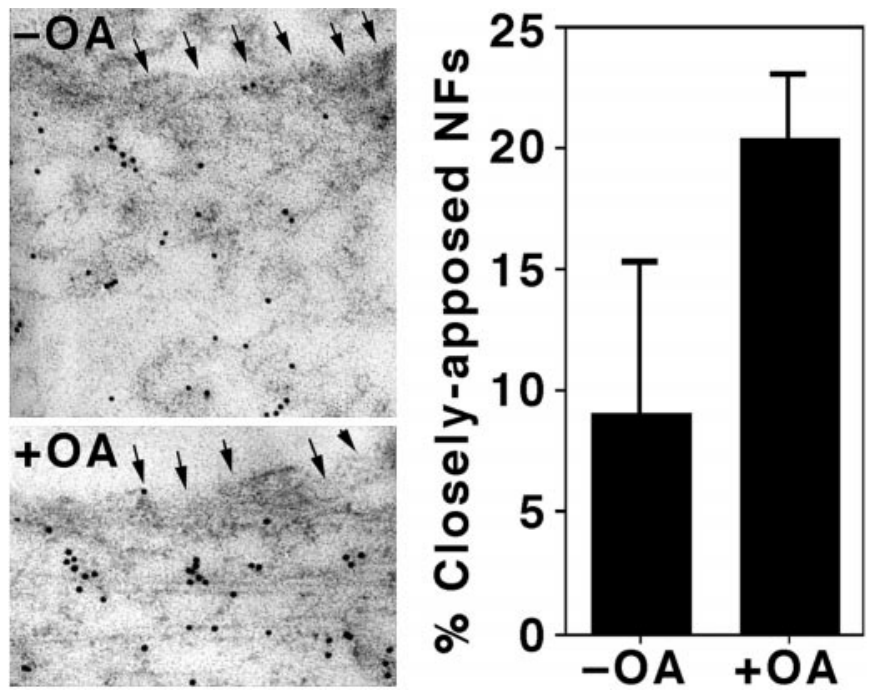

Figure 3. The phosphatase inhibitor okadaic acid $(O A)$ increases NF-NF associations within axonal neurites. The panels present peripheral areas of axonal neurites from OA-treated and untreated cells that were probed with SMI-31, followed by colloidal gold-conjugated secondary antibody; the plasma membrane is noted by arrows. The accompanying graph presents quantification of the percentage of total SMI-31-immunoreactive filaments observed within $20 \mathrm{~nm}$ of another SMI-31-immunoreactive filament in longitudinally oriented sections. Note the more than twofold increase in closely apposed NFs after OA treatment ( $p<0.05$; Student's $t$ test).

oriented NF bundles (17 $\pm 1.7 \mathrm{~nm}$; see above). We performed these analyses on peripheral areas (i.e., close to the plasma membrane) rather than attempting to quantify any OA-induced alterations in the size of the central NF bundle, because the overall axonal caliber, as well as the size of central NF bundles, varied from cell to cell. These analyses revealed a more than twofold increase $(p<0.05)$ in closely apposed NFs (Fig. 3). This OA-mediated increase in NF-NF association was unlikely to be derived artifactually solely from an increase in total axonal phospho-NFs (Shea et al., 1993) because, even after OA treatment, NFs occupied a very small percentage of the axonal area within thin sections of peripheral axonal regions (Fig. 3). These findings demonstrate a functional relationship between NF phosphorylation and NF bundling, one interpretation of which is that NF phosphorylation mediates NF bundling.

\section{Transporting NF subunits exhibit a delay in association with bundled NFs}

Because more extensive C-terminal NF-H phosphorylation has been associated with slower-moving NFs (Lewis and Nixon, 1988; Jung et al., 2000), we hypothesized that individual and bundled NFs could be distinguished by differential transport and/or subunit turnover. This hypothesis was tested by microinjection of biotinylated NF subunits (Fig. 4A,B), transfection with a construct encoding GFP-tagged NF subunits (Fig. 4C,D), and pulsechase metabolic radiolabeling of endogenous NF subunits.

As in previous immunofluorescent analyses (Jung et al., 1998; Yabe et al., 1999), immuno-EM analyses revealed that biotinylated subunits were detected along the entire axonal length by $2 \mathrm{hr}$ after their injection into perikarya. However, the resolution afforded by these ultrastructural analyses revealed that biotinylated subunits were distributed differentially among axonal NFs at early versus later times after injection. Despite their distribution along the entire axonal length within $2 \mathrm{hr}$, biotinylated subunits

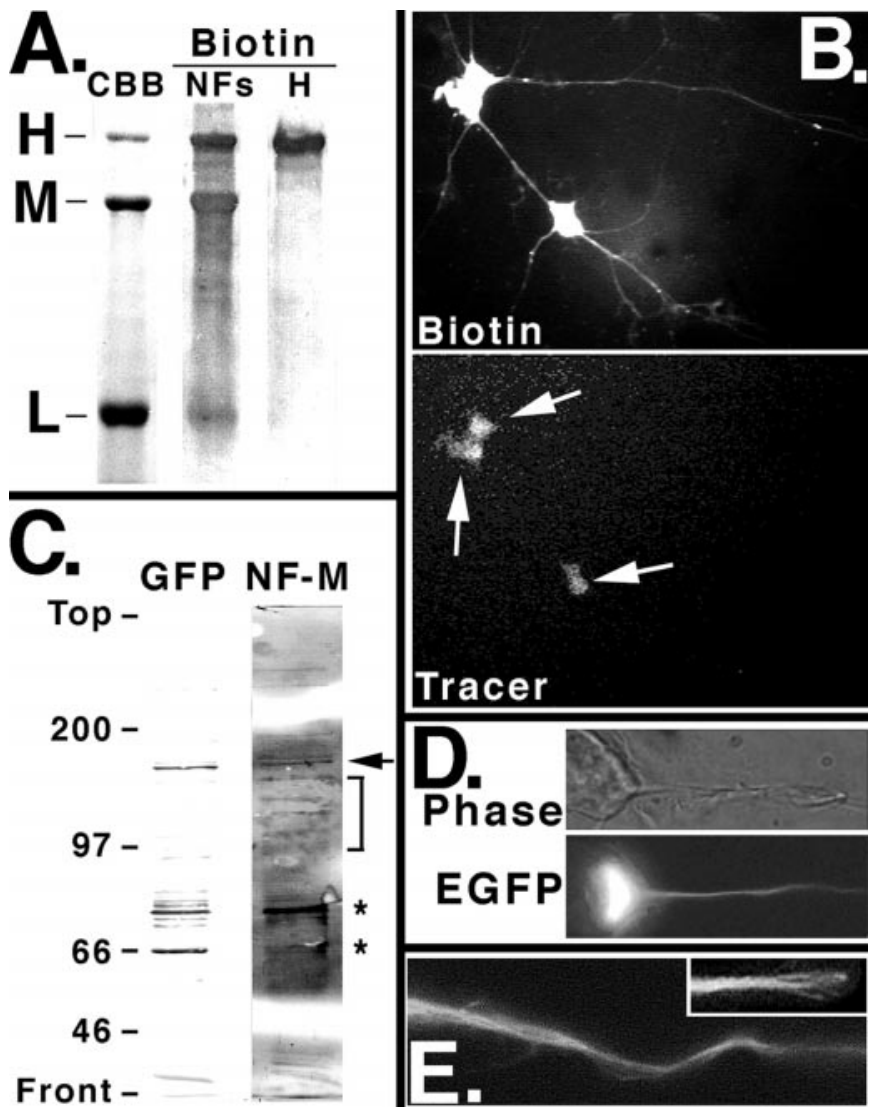

Figure 4. Biotinylated and GFP-tagged NF subunits and their intracellular distribution after microinjection and transfection. $A$, Coomassie blue staining after SDS-gel electrophoresis of NFs isolated from bovine spinal cords $(C B B)$ and immunoblot analysis of this preparation $(N F s)$ after biotinylation and staining of chromatographically separated NF-H and NF-L, as indicated. $B$, UV images of cells $2 \mathrm{hr}$ after the injection of biotinylated NF-H (Biotin) and fluorescein-conjugated tracer (Tracer), as indicated. $C$, Immunoblot analyses of Triton-insoluble cytoskeletons from cells stably transfected with the eGFP-NF-M construct. In addition to NF-M isoforms migrating between 97 and $145 \mathrm{kDa}$ detected by antiNF-M antibodies (bracket), note the presence of an NF-M-reactive isoform migrating at $\sim 170 \mathrm{kDa}$ that also is detected by anti-GFP antibodies (arrow). Asterisks indicate lower-molecular-weight C-terminal proteolytic products derived from NF-M; note the labeling of these products by both GFP and NF-M antibodies. Identity of these species as breakdown products was confirmed by their increase at the expense of full-length eGFPNF-M after the incubation of additional samples at room temperature for 30-60 min (data not shown). D, Fluorescent and corresponding phasecontrast image of an unextracted, transiently transfected cell. E, The axonal neurite of a transiently transfected cell processed under conditions that promote the splaying of axonal NFs (Brown, 1998). The resultant loosening of bundled NFs confirms the filamentous nature of axonal GFP fluorescence. Inset presents the distal region, just before the growth cone, of a second axonal neurite.

remained concentrated along the periphery of the axon with respect to its longitudinal axis and displayed relatively little localization within NF bundles (Fig. 5). This phenomenon was not derived by steric inhibition of antibody penetration, because, as shown above, SMI-31 immunoreactivity was distributed evenly among bundled and nonbundled axonal filaments at all of the times that were examined, and RT97 immunoreactivity was even more prominent on bundled versus nonbundled NFs (see Fig. 1). Moreover, biotinylated subunits underwent progressive association with NFs in bundles along the axon and were distributed relatively evenly among bundled and nonbundled NFs throughout the axon by $18 \mathrm{hr}$ after microinjection (Fig. 5). 
Figure 5. Ultrastructural analyses of the distribution of microinjected NF subunits. The panels present immuno-EM analyses of the distribution of biotinylated NF-H after its microinjection into differentiated NB2a/d1 cells, as described in Materials and Methods. Top, Biotinylated subunits (arrows) were readily localized along peripherally situated NFs within central axonal segments within 2 hr after injection but were excluded in large part from the bundle (indicated by arrowheads along the right side of the micrographs). By $18 \mathrm{hr}$, however, biotinylated subunits were dispersed throughout the bundles. The accompanying graph presents the relative distribution of biotinylated NF-H subunits within individual and bundled NFs at 2, 6 , and $18 \mathrm{hr}$ after injection; note the progressive association of biotinylated subunits with bundled NFs. Bottom, Comparative analyses of the distribution of biotinylated $\mathrm{N}-\mathrm{H}$ subunits within bundles and individual NFs within proximal and distal regions of the axonal shaft at 2 and $6 \mathrm{hr}$ after microinjection; for these analyses we analyzed regions within the proximal and distal halves of the axonal shaft, excluding the hillock and the growth cone. Note the presence of some gold particles (arrows) within the centrally situated bundle (denoted by arrowheads along right side of the micrograph) within proximal segments at $2 \mathrm{hr}$ after injection versus their absence in distal segments as well as in central segments (e.g., $2 \mathrm{hr}$ micrograph in top panel). Note that not all NFs within bundles display even labeling within the proximal shaft at $2 \mathrm{hr}$; some NFs apparently remain unlabeled. The accompanying graph presents the relative distribution of biotinylated NF-H subunits within bundles in proximal and distal axonal shafts at 2 and $6 \mathrm{hr}$ after injection; note that the vast majority of bundleassociated biotinylated subunits is localized within the proximal half of the axonal shaft at $2 \mathrm{hr}$ after injection but is distributed equally between proximal and distal regions by $6 \mathrm{hr}$ after injection.

We next considered the mechanism(s) by which biotinylated NF subunits associated with NF bundles. Should bundled NFs undergo axonal transport, we would expect biotinylated subunits to accumulate initially, or in greater concentration, at the proximal-most end of the bundle and to do so only later along the more distal portion of bundled NFs. By contrast, should bundled NFs represent essentially nonmoving NFs (e.g., "stationary" NFs; Nixon and Logvinenko, 1986) and should NFs in these bundles be replaced by subunit exchange (Takeda et al., 1994), we would expect biotinylated subunits to accumulate simultaneously at similar concentrations along the length of the bundle. This latter possibility is feasible because immunofluorescent analyses (Jung et al., 1998) (see also Fig. 4B) demonstrated that biotinylated subunits were present at similar concentrations along the entire axonal length substantially before their association within bundled NFs, as revealed herein by ultrastructural analyses (Fig. 5). We therefore compared the distribution of biotinylated NF-H within segments from the proximal half of the shaft (taking care to exclude the hillock and most proximal segment) with segments from the distal half of the shaft (taking care to exclude the distal-most segment and the growth cone). Biotinylated subunits in proximal and distal segments of the axonal shaft were scored as associated with bundles when they appeared on or adjacent to bundles (Fig. 5) or otherwise were considered not to be associated with bundles. At $2 \mathrm{hr}$ after injection $76 \%$ of those biotinylated subunits that were associated with bundles were confined to the proximal portion of bundles. By $6 \mathrm{hr}$ after injection, however, those biotinylated subunits that were associated with bundles were distributed evenly between the proximal and distal aspects of bundles (Fig. 5). Not all NFs within bundles displayed even labeling within the proximal shaft at $2 \mathrm{hr}$, and some NFs remained unlabeled (Fig. 5); only $45 \pm 14 \%$ of NFs within bundles was labeled at this early time $(n=5$ axons). By contrast, nearly all filaments were labeled with anti-biotin by $18 \mathrm{hr}$ (Fig. 5). This delay was not attributable merely to steric inhibition of newly transporting subunits entering bundles, because in some sections the center of the bundle was labeled more strongly than its edges (see Prox. Shaft at 2 hr; Fig. 5). Although these findings do not exclude the possibility that biotinylated subunits exchange with preexisting NFs in bundles, the observed anterograde progression of the appearance of biotinylated subunits within bundles suggests that bundled NF also may undergo transport, albeit more slowly than nonbundled NFs. This conclusion is supported further by our use of biotinylated NF-H rather than NF-L in these ultrastructural analyses, because NF-H would have been more likely to undergo more rapid exchange than NF-L (Takeda et al., 1994).

The temporal distribution of newly transporting subunits also 

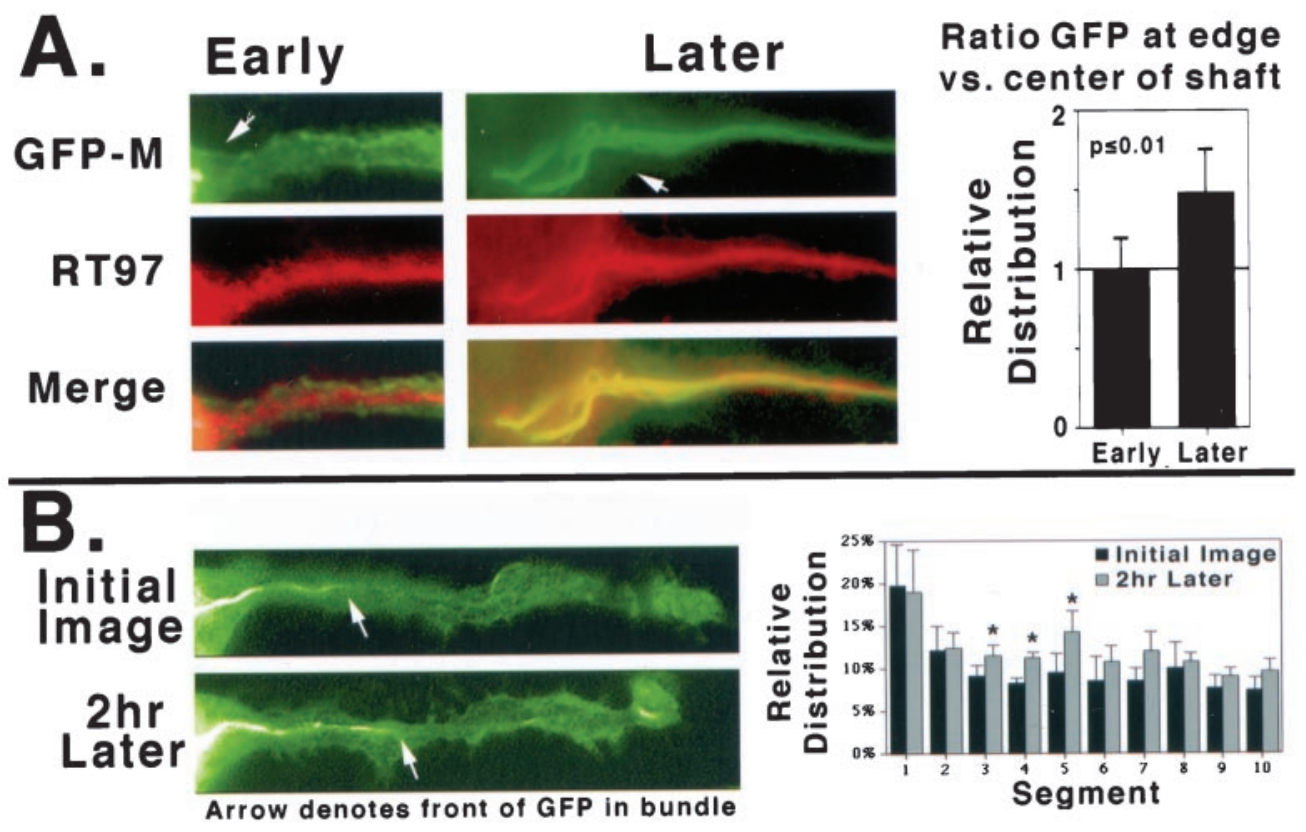

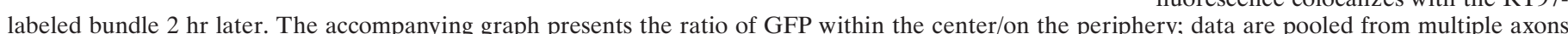

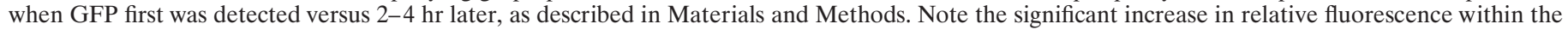

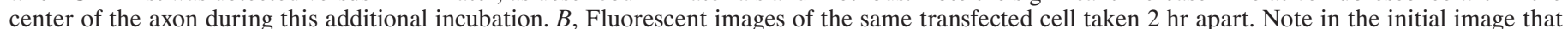

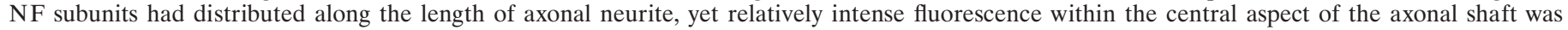

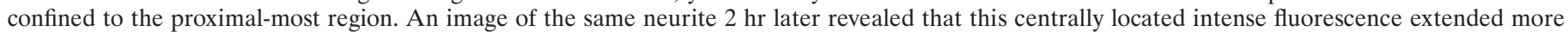

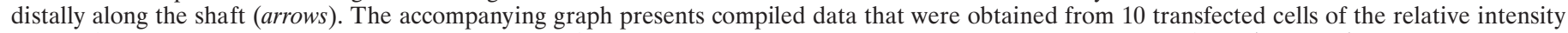

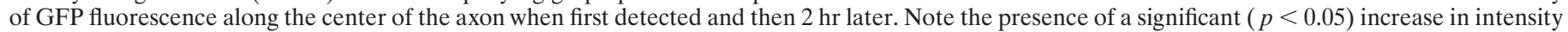
within central segments in this $2 \mathrm{hr}$ interval. was probed by the transfection of differentiated cells with a construct encoding GFP-tagged NF-M (Yabe et al., 1999). After overnight incubation to allow for the accumulation of GFP-tagged subunits (Yabe et al., 1999), the cells were fixed and processed for RT97 immunoreactivity. As with microinjected biotinylated subunits, GFP-tagged NF-M initially was observed along the periphery of axonal neurites and did not colocalize with the more centrally situated RT97 immunoreactivity (Fig. 6A). After an additional 2-4 hr of incubation, however, prominent GFP fluorescence colocalized with RT97 immunoreactivity (Fig. 6A). These data indicate that newly transporting NF subunits exhibit a delayed association with bundled NFs.

Auto-fluorescence of GFP-tagged subunits precluded the necessity of fixation before observation and therefore also afforded the ability to observe dynamic alteration in the distribution of newly transporting NF subunits repeatedly within the same axonal neurites. Such analyses demonstrated that NF subunits had distributed along the length of axonal neurites within $2 \mathrm{hr}$ (Fig. $6 B$ ). In addition, relatively intense fluorescence was observed within the central aspect of the proximal-most region of the axonal shaft. Subsequent observations of the same axonal neurite revealed this centrally located intense fluorescence to undergo a progressive proximal-distal accumulation (Fig. 6B). The intense centrally situated GFP fluorescence is consistent with the relatively large number of NFs within the centrally situated bundle as observed in ultrastructural analyses and in RT97 immunofluorescence (see above). These data are consistent with our above speculation that bundled NFs indeed undergo axonal transport but do so more slowly than do peripherally situated individual NFs. These data do not, however, exclude the possibility that
Figure 6. GFP-tagged NF subunits exhibit a delayed association with bundled NFs. $A$, Images of cells transfected 16-24 hr previously with eGFP-NF-M and then fixed and immunostained with RT97. Arrowheads in the GFP images denote the neurite hillock. As described in Materials and Methods (see also Yabe et al., 1999), 16-24 hr of further incubation was required after transfection for the accumulation of sufficient eGFP-tagged NF-M for detection. Cells were fixed either as soon as GFP immunofluorescence was detected (Early) within axons or 2-4 hr later (Later). GFP fluorescence initially was observed along the periphery of axonal neurites and did not colocalize with RT97. Colocalization of GFP and RT97 is revealed by yellow-orange immunofluorescence in merged images. Note that GFP fluorescence initially is concentrated along the periphery of axonal neurites and does not exhibit appreciable colocalization with the centrally situated RT97-labeled bundle. By contrast, GFP fluorescence colocalizes with the RT97-

subunit and/or filament exchange also occurs along the length of bundled NFs.

The distribution of newly transported NF subunits also was monitored by pulse-chase radiolabeling. We reasoned that, if bundled NFs undergo relatively slower transport/turnover than do individual NFs, then radiolabeled subunits initially would be recovered predominantly within individual NFs and only later would be associated with bundled NFs. Cultures were radiolabeled with $\left[{ }^{35} \mathrm{~S}\right]$ methionine for $15 \mathrm{~min}$, followed by replacement of the medium with fresh medium lacking radiolabel, and were incubated for a total of $4 \mathrm{hr}$ (Shea et al., 1990). Cultures were harvested at these times and fractionated under conditions that separate axonal neurites from perikarya (Shea et al., 1993). These fractions, respectively, were fractionated further to generate Triton-soluble and Triton-insoluble material. Finally, Tritoninsoluble material from axonal neurites was sedimented over a 1 M sucrose cushion to separate bundled and individual NFs (see Fig. 3). As in previous studies (Shea et al., 1990) radiolabeled NF subunits were associated with axonal Triton-insoluble structures within $15 \mathrm{~min}$. However, virtually all detectable radiolabeled subunits were retained by the sucrose cushion at this time (Fig. 7). By contrast, when cultures were harvested after $4 \mathrm{hr}$ in the absence of additional radiolabel, virtually all radiolabeled subunits were recovered within the material that sedimented through the sucrose cushion. Because bundled but not individual NFs sedimented through this cushion (see Fig. 2), these findings indicate that radiolabeled subunits exhibited a delayed association with bundled NFs and therefore suggest that bundled NFs undergo slower turnover than do individual NFs within axonal neurites. 
Figure 7. Endogenous axonal NF subunits exhibit a delayed association with NF bundles. The panels present immunoblot and autoradiographic analyses of cytoskeletons derived from perikaryal (Soma) and axonal preparations from cells that were pulselabeled for $15 \mathrm{~min}$ and immediately harvested or cultures from which radiolabeled medium was replaced with medium lacking radiolabel and in which incubation was continued for a total of $4 \mathrm{hr}$, as indicated. Axonal material recovered at the interface and material that sedimented through a $1 \mathrm{M}$ sucrose cushion (pellet) are indicated. The relative migration of molecular weight stan-

dards is indicated on the left side of the figure. Immunoreactive and immunoprecipitated species corresponding to NF-H, NF-M, and NF-L are indicated also. The accompanying immunoblots (probed with R39) confirm the presence of NF subunits in cytoskeletons derived from all fractions. Note that radiolabeled NF subunits are associated with perikaryal and axonal NFs within 15 min of radiolabeling, yet within axonal neurites are recovered virtually entirely from the interface and not from the pellet. Note further that by $4 \mathrm{hr}$ the majority of radiolabeled subunits is associated with the pellet. Because bundled NFs sedimented through sucrose under these conditions yet individual NFs were retained at the interface, these data indicate that NF subunits associate with individual NFs within axons before their association with bundled NFs.

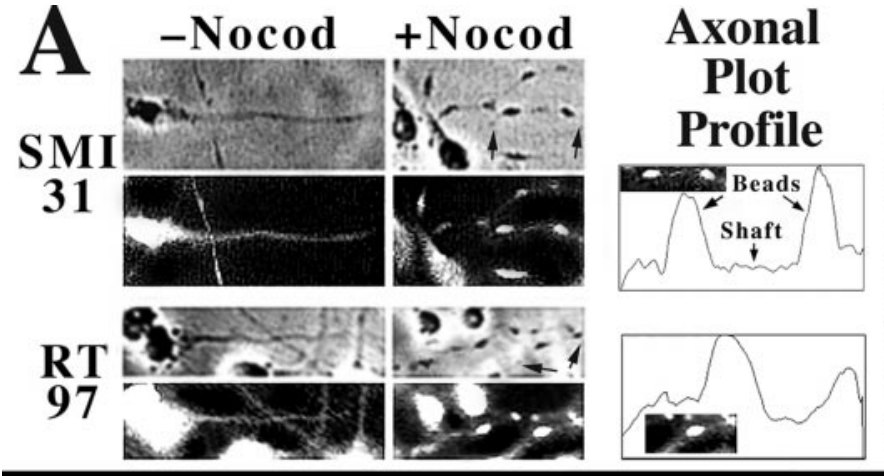

B Phase
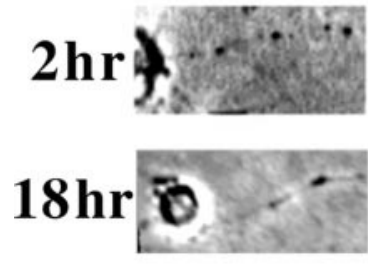
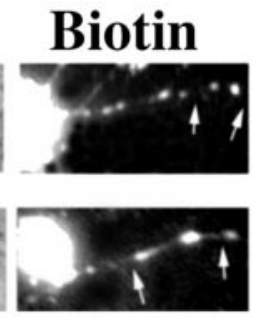

Immunoblot

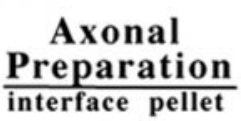

\section{Soma \\ $15 \mathrm{~min}$ \\ Pulse}
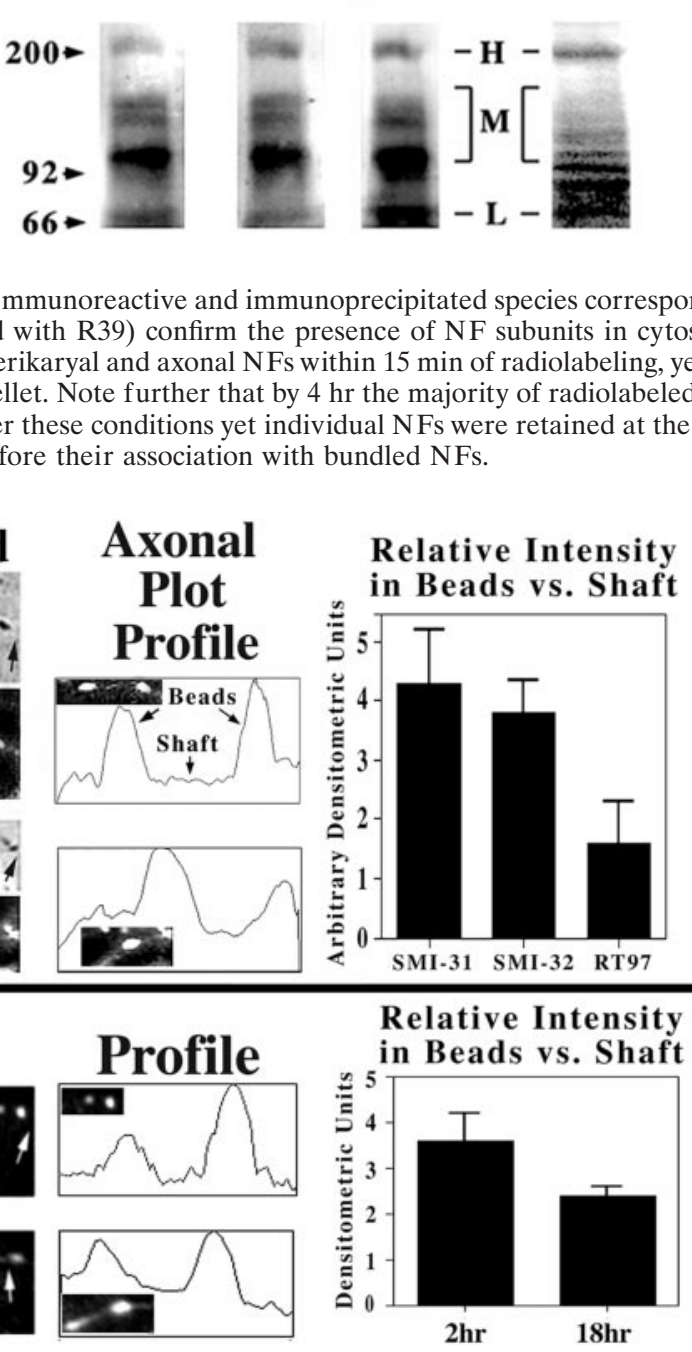

Figure 8. Nocodazole alters axonal neurite morphology and the distribution of axonal NF immunoreactivity. $A$, Immunofluorescent analyses of the distribution of SMI-31 and RT97 immunoreactivity with and without treatment with nocodazole for $2 \mathrm{hr}$, as indicated. Corresponding phase-contrast images are presented; note that nocodazole induces overall thinning of the axonal shaft (white arrows) with periodic varicosities or beads. The accompanying plot profiles present the distribution of NF immunoreactivity within select regions of axonal neurites; these regions are presented as insets within the graphs and are indicated by arrows in the appropriate phase-contrast image. Note that relatively more RT97 immunoreactivity is retained within shafts than is SMI-31 immunoreactivity. The accompanying bar graph presents a comparison of the relative distribution of NF immunoreactivity within varicosities versus adjacent areas of the shaft; SMI-32 immunoreactivity (not presented in micrographs) also was included in these analyses. Note that approximately fourfold more SMI-31 and SMI-32 immunoreactivity was localized within varicosities as opposed to the adjacent areas of the axonal shaft, whereas only $\sim 1.5$-fold more RT97 immunoreactivity distributed within varicosities as opposed to the shaft. $B$, Biotin immunoreactivity in cells treated with nocodazole at 2 and $18 \mathrm{hr}$ after injection, as indicated. Corresponding phase-contrast images are presented also. The accompanying plot profiles present the distribution of biotin immunoreactivity within select regions of axonal neurites; these regions are presented as insets within the graphs and are indicated by arrows within the immunofluorescent images. Note that relatively more immunoreactivity is retained within shafts at 18 hr after injection. The accompanying bar graph presents the relative distribution of biotin immunoreactivity within varicosities versus adjacent areas of the shaft. Note that, when microinjected cells were treated with nocodazole at $2 \mathrm{hr}$ after injection, $\sim 3.5$-fold more biotin immunoreactivity distributed within varicosities versus adjacent areas of the shaft. Conversely, only approximately twofold more biotin immunoreactivity partitioned within beads versus shafts when nocodazole treatment was performed $18 \mathrm{hr}$ after injection.

\section{Bundled NFs are relatively resistant to perturbation in situ}

Finally, should bundled NFs indeed represent population(s) of NFs that are undergoing relatively more NF-NF interactions in situ than those that are situated more peripherally, the individual $\mathrm{NFs}$, we reasoned that bundled NFs may be more resistant to treatments that induce perturbation of the axonal cytoskeleton. Previous studies have demonstrated differential distribution of axonal constituents after the stretching of axonal fibers (Ochs et al., 1989). Significant NF immunoreactivity was dispersed into the bead-like swellings, or varicosities, that were generated along the axon after experimental stretching, whereas longitudinal filamentous profiles remained within the compressed center of the axonal shaft (Ochs et al., 1989). Although this stretching technique is not directly applicable to cultured neuronal cells, a similar morphological profile is generated after short-term treatment of NB2a/d1 cells with microtubule-depolymerizing drugs such as colchicine and nocodazole (Shea and Beermann, 1994; Jung et al., 1998). Surviving neurites exhibit an overall thinning in caliber and exhibit periodic varicosities along their length (Fig. 8) (see also Shea and Beermann, 1994). We therefore considered that nocodazole treatment may foster differential distribution of axonal NFs in a manner analogous to that observed after the stretching of fibers. Should bundled NFs represent a population of NFs that have undergone increased NF-NF associations, we reasoned that they would tend to remain centrally located along 
the axonal shaft, whereas individual NFs may distribute more readily within resultant varicosities. To test this possibility, we examined the distribution of SMI-31, SMI-32, and RT97 immunoreactivity after treatment of differentiated NB2a/d1 cells with nocodazole for $2 \mathrm{hr}$ (Fig. $8 \mathrm{~A}$ ). Immunoreactivity toward these antibodies partitioned differentially within resultant beads and thinned areas of the axonal shaft. Approximately fourfold more SMI-31 and SMI-32 immunoreactivity was localized within varicosities as opposed to adjacent areas of the axonal shaft. By contrast, only $\sim 1.5$-fold more RT97 immunoreactivity distributed within varicosities as opposed to the shaft. These data suggest that RT97 selectively labels a population of NFs that are relatively resistant to redistribution. Because RT97 selectively labels bundled NFs, these data support the interpretation that bundled NFs are relatively more stable than individual NFs in situ. This possibility was probed further by monitoring the distribution of microinjected NF-H within axonal neurites treated as above with nocodazole at $2 \mathrm{hr}$ after injection (at which time the majority of injected subunits is associated with individual, peripherally situated NFs) and at $18 \mathrm{hr}$ after injection (by which time some injected subunits have associated with bundled NFs). We reasoned that, should bundled NFs indeed be more resistant than individual NFs to redistribution after nocodazole treatment, biotinylated subunits should localize within nocodazole-induced varicosities to a greater extent at $2 \mathrm{hr}$ after injection than at $18 \mathrm{hr}$ after injection. When microinjected cells were treated with nocodazole at $2 \mathrm{hr}$ after injection, $\sim 3.5$-fold more biotin immunoreactivity distributed within varicosities versus adjacent areas of the shaft. Conversely, only approximately twofold more biotin immunoreactivity partitioned within beads versus shafts when nocodazole treatment was performed $18 \mathrm{hr}$ after injection (Fig. $8 B$ ). These findings indicate that a portion of microinjected subunits attained increased resistance to nocodazole-induced redistribution at a time by which some subunits have associated with bundled NFs and suggest that bundled NFs are indeed relatively more stable than individual NFs in situ.

\section{DISCUSSION}

We present evidence herein that axonal NFs consist of at least two populations that can be distinguished on the basis of differential NF-NF associations and C-terminal phosphorylation. NFs rich in site-specific $\mathrm{C}$-terminal phosphorylation were organized preferentially into bundles. When we monitored the spatial and temporal distribution of newly transported subunits by microinjection, transfection, and radiolabeling, our analyses further demonstrate that bundled NFs either do not undergo transport or undergo transport at a rate slower than that of individual NFs.

Differential transport of individual versus bundled NFs may represent the morphological equivalent of the broadening of the transport wave routinely observed for radiolabeled NF subunits in situ (Nixon and Logvinenko, 1986; Watson et al., 1989; Lasek et al., 1992, 1993; Jung and Shea, 1999). Retardation of the transport of bundled NFs is likely to result from a preponderance of phosphate-dependent and/or other physical filament-filament interactions among the bundled NFs (Leterrier and Eyer, 1987; Shaw and Hou, 1990; Gotow and Tanaka, 1994; Nagakawa et al., 1995; Leterrier et al., 1996). Previous studies demonstrated that NFs move apart on disruption of axonal membrane integrity, indicating that such NFs are not cross-linked physically (Brown and Lasek, 1993); however, these investigators pointed out that their findings remained consistent with the data of Shaw and Hou (1990) in which some NFs were recovered as bundles. Our recov- ery in cytoskeletal preparations of both individual and bundled NFs, with distinct phospho-dependent immunoreactivity, supports and unifies the conclusions of these previous studies. We consider it likely that peripherally situated NFs and centrally situated bundled NFs represent a continuum in situ rather than discrete populations and that individual NFs within these experimentally separable categories are also likely to display broad ranges of transport and turnover rates. This possibility is supported by the demonstration that phosphorylated and nonphosphorylated NFs can exist side by side within axons of cultured neurons (Brown, 1998). Further studies directed toward elucidating any potential differences among NFs within these two broadly characterized "populations" are warranted.

If, as our data and that of previous studies suggest, bundled NFs represent a population that is undergoing relatively more $\mathrm{NF}-\mathrm{NF}$ interactions than are individual NFs, these findings are consistent with the notion that NF-NF interactions may compete with the interactions of NFs with their transport motor and foster increased residence time of some NFs within axons (Lasek et al., 1992; Nixon, 1993, 1998). This interpretation is also consistent with the observation that NFs containing the most highly phosphorylated NF-H variants undergo axonal transport at the slowest rates (Lewis and Nixon, 1988), whereas poorly phosphorylated NFs travel at faster rates (Jung et al., 2000) and with the observed slowing of NF axonal transport and increase in C-terminal NF phosphorylation after the inhibition of phosphatase activity in situ (Jung et al., 1999). In this regard, newly transporting NF subunits are associated in situ either directly or indirectly with the motor protein kinesin (Yabe et al., 1999, 2000). Furthermore, this association is disrupted by site-specific C-terminal NF phosphorylation such that RT97-immunoreactive NF subunits do not interact with kinesin (Yabe et al., 2000). These findings are consistent with the interpretation that bundled NFs (which are preferentially reactive with RT97) are associated less frequently with their motor complex than are individual NFs (for review, see Shea and Yabe, 2000). Relatively rapid translocation of some NF subunits along the axonal length (i.e., individual NFs) is consistent with in vivo observation of the transport of radiolabeled NF subunits at rates 100 times greater than the bulk of translocating subunits (Lasek et al., 1993). Observation of faster-moving individual NFs and slower-moving bundled NFs is also consistent with the recent demonstration by Wang et al. (2000) of the rapid axonal transport of NFs interrupted by pauses. In these analyses substantial gaps were observed in the axonal NF array. These gaps as a whole did not translocate, although some NFs traversed the gaps, demonstrating NF transport over a broad range of rates. It is possible that the faster-moving NFs correspond to the individual NFs in our analyses, whereas the slowermoving NFs (i.e., surrounding the gaps) correspond to the bundled NFs in our analyses. The periodic pausing exhibited by the faster-moving NFs may correspond further to a period of limited interaction with other NFs that could, if prolonged sufficiently, lead to bundling. Further investigations in this regard will be of interest.

Some NF subunits enter axons and undergo transport while still in Triton-soluble form (Jung et al., 1998), and such subunits include precursors for NF assembly (Shea et al., 1990, 1997; Shea, 1994; Jung et al., 1998; Yabe et al., 1999). Although our findings of differential transport rates for axonal NFs are in agreement with existing polymer transport models (for review, see Baas and Brown, 1997), they also leave open the possibility that monomeric/oligomeric NF-H subunits selectively may incorporate at 
least to some degree into preexisting NFs and/or assemble onto the ends of elongating NFs. Several lines of evidence support this possibility, including (1) detection of NF-H immunoreactivity by immuno-EM analyses in the absence of any filamentous profiles within neurons in situ (Gotow and Tanaka, 1994); (2) transport of monomeric/oligomeric NF-M in the absence of axonal NFs (Terada et al., 1994); (3) slow axonal transport of some NF subunits in punctate, apparently nonfilamentous form (Yabe et al., 1999); and (4) selective accumulation of newly transported NF-H into Triton-insoluble structures within the distal-most segment of growing axons (Yabe et al., 1997). Incorporation of NF subunits into bundled NFs may occur via exchange of individual NFs and/or subunits along the length of bundled NFs. Our observation of stronger labeling of proximal bundled NFs before distal NFs leads us to speculate that the bundle is indeed translocating, albeit more slowly than surrounding individual NFs. However, whether bundled NFs undergo transport or not does not exclude the possibility that the exchange of NF subunits still may occur along the length of bundled NFs.

Controversy exists regarding three major aspects of NF biology: (1) whether axonal NFs constitute a single population or multiple populations, (2) whether NFs are cross-linked in situ, and (3) whether axonal NFs undergo turnover within the axonal cytoskeleton by replacement with new NFs or instead by incorporation of newly translocating subunits into existing, nonmoving NFs. To the extent that one can extrapolate findings in a cell culture model to the situation in situ, the data of the present study address each of these controversies. In summary, our data support the interpretations that axonal NFs consist of multiple populations that undergo axonal transport although over a wide range of rates and that some, but not all, NFs apparently are cross-linked physically in situ. The data at present do not allow us to resolve whether axonal NFs undergo replacement by newly transporting polymers, by subunit exchange, or by both. The two NF populations observed in these growing axons may represent the developmental precursor of a stationary population of NFs that is replaced along its length by newly transported NFs (Nixon, 1993, 1998). Furthermore, establishment of a stationary cytoskeleton may favor the exchange of NF subunits with nonmoving NFs as a major mode of NF turnover (Hirokawa et al., 1997). Although such a scenario in essence can be envisioned as a logical developmental exaggeration of the NF populations observed herein, clarification of this issue requires further investigation of $\mathrm{NF}$ dynamics in situ.

\section{REFERENCES}

Anderton BH, Breinburg D, Downes MJ, Green PJ, Tomlinson BE, Ulrich J, Wood JN, Kahn J (1982) Monoclonal antibodies show that neurofibrillary tangles and neurofilaments share antigenic determinants. Nature 298:84-86.

Baas PW, Brown A (1997) Slow axonal transport: the polymer transport model. Trends Cell Biol 7:380-384.

Brady ST (1993) Motor neurons and neurofilaments in sickness and health. Cell 73:1-3.

Brown AT (1997) Visualization of single neurofilaments by immunofluorescence microscopy of splayed axonal cytoskeletons. Cell Motil Cytoskeleton 38:133-145.

Brown AT (1998) Contiguous phosphorylated and nonphosphorylated domains along axonal neurofilaments. J Cell Sci 111:455-467.

Brown A, Lasek RJ (1993) Neurofilaments move apart freely when released from the circumferential constraint of the axonal plasma membrane. Cell Motil Cytoskeleton 26:313-324.

Chin SSM, Liem RKH (1989) Expression of rat neurofilament proteins NF-L and NF-M in transfected non-neuronal cells. Eur J Cell Biol 50:475-490.

Galbraith JA, Reese TS, Schlief ML, Gallant PE (1999) Slow transport of unpolymerized tubulin and polymerized neurofilament in the squid giant axon. Proc Natl Acad Sci USA 96:11589-11594.
Gotow T, Tanaka J (1994) Phosphorylation of neurofilament H subunit as related to arrangement of neurofilaments. J Neurosci Res 37:691-713.

Hirokawa N, Terada S, Funakoshi T, Takeda S (1997) Slow axonal transport: the subunit transport model. Trends Cell Biol 7:384-388.

Jung C, Shea TB (1999) Regulation of neurofilament axonal transport by phosphorylation in optic axons in situ. Cell Motil Cytoskeleton 43:230-240.

Jung C, Yabe J, Wang F-S, Shea TB (1998) Neurofilaments are present within axonal neurites before incorporation into Triton-insoluble structures. Cell Motil Cytoskeleton 40:44-58.

Jung C, Yabe JT, Lee S, Shea TB (2000) Hypophosphorylated neurofilament subunits undergo axonal transport more rapidly than more extensively phosphorylated subunits in situ. Cell Motil Cytoskeleton 47:120-129.

Koehnle TJ, Brown A (1999) Slow axonal transport of neurofilament protein in cultured neurons. J Cell Biol 144:447-458.

Lasek RJ (1986) Polymer sliding in axons. J Cell Sci Suppl 5:161-179.

Lasek RJ, Paggi P, Katz MJ (1992) Slow axonal transport mechanisms move neurofilaments relentlessly in mouse optic axons. J Cell Biol 117:607-616.

Lasek RJ, Paggi P, Katz MJ (1993) The maximum rate of neurofilament transport in axons: a view of molecular transport mechanisms continuously engaged. Brain Res 616:58-64.

Leterrier J-F, Eyer J (1987) Properties of highly viscous gels formed by neurofilaments in vitro. A possible consequence of specific interfilament cross-bridging. Biochem J 245:93-101.

Leterrier J-F, Käs J, Hartwig J, Vegners R, Janmey P (1996) Mechanical effects of neurofilament cross-bridges. J Biol Chem 271:15687-15694.

Lewis SE, Nixon RA (1988) Multiple phosphorylated variants of the high molecular mass subunit of neurofilaments in axons of retinal cell neurons: characterization and evidence for their differential association with stationary and moving neurofilaments. J Cell Biol 107:2689-2701.

Nagakawa T, Chen J, Zhang Z, Kanai Y, Hirokawa N (1995) Two distinct functions of the carboxyl terminal tail domain of NF-M upon neurofilament assembly: cross-bridge formation and longitudinal elongation of filaments. J Cell Biol 129:411-429.

Nixon RA (1993) The regulation of neurofilament protein dynamics by phosphorylation: clues to neurofibrillary pathology. Brain Pathol 3:29-38.

Nixon RA (1998) The slow transport of cytoskeletal proteins. Curr Opin Cell Biol 10:87-92.

Nixon RA, Lewis SE (1986) Differential turnover of phosphate groups on neurofilament subunits in mammalian neurons in vivo. J Biol Chem 261:16298-16301.

Nixon RA, Logvinenko KB (1986) Multiple fates of newly synthesized neurofilament proteins: evidence for a stationary neurofilament network distributed nonuniformly along axons of retinal ganglion cell neurons. J Cell Biol 102:647-659.

Ochs S, Jersild RA, Li J-M (1989) Slow transport of freely movable cytoskeletal components shown by beading partition of nerve fibers in the cat. Neuroscience 33:421-430.

Sambrook J, Fritsch EF, Maniatis T (1989) Molecular cloning, a laboratory manual, 2nd Ed. Cold Spring Harbor, NY: Cold Spring Harbor.

Shaw G, Hou Z-C (1990) Bundling and cross-linking of intermediate filaments of the nervous system. J Neurosci Res 25:561-568.

Shea TB (1990) Transient increase in vimentin in axonal cytoskeletons during differentiation in NB2a/d1 cells. Dev Brain Res 521:343-346.

Shea TB (1994) Triton-soluble phosphovariants of the high molecular weight neurofilament subunit from NB2a/d1 cells are assembly-competent: implications for normal and abnormal neurofilament assembly. FEBS Lett 343:131-136.

Shea TB (1999) Selective stabilization of microtubules within the proximal region of developing axonal neurites. Brain Res Bull 48:255-261.

Shea TB, Beermann ML (1994) Sequential requirements for NFs, MTs, and the MT-associated proteins MAP1B and tau in axonal neurite initiation, elongation, and stabilization. Mol Cell Biol 5:863-875.

Shea TB, Yabe JT (2000) Occam's razor slices through the mysteries of neurofilament axonal transport: can it really be so simple? Traffic 1:522-523.

Shea TB, Sihag RK, Nixon RA (1988) Neurofilament triplet proteins of NB2a/d1 neuroblastoma: post-translational modification and incorporation into the cytoskeleton during differentiation. Dev Brain Res 43:97-109.

Shea TB, Beermann ML, Nixon RA (1989) Appearance and localization of phosphorylated variants of the high molecular weight neurofilament protein in NB2a/d1 cytoskeletons during differentiation. Dev Brain Res 50:142-146.

Shea TB, Sihag RK, Nixon RA (1990) Dynamics of phosphorylation and assembly of the high molecular weight NF subunit in NB2a/d1 neuroblastoma. J Neurochem 55:1784-1792.

Shea TB, Fischer I, Paskevich PA, Beermann ML (1993) The protein phosphatase inhibitor okadaic acid increases axonal NFs and neurite caliber, and decreases axonal MTs in NB2a/d1 cells. J Neurosci Res 35:507-521. 
Shea TB, Dahl D, Nixon RA, Fischer I (1997) Triton-soluble phosphovariants of the heavy neurofilament subunit in developing and mature mouse central nervous system. J Neurosci Res 48:515-523.

Straube-West K, Loomis PA, Opal P, Goldman RD (1996) Alterations in neuronal intermediate filament organization, functional implications, and the induction of pathological changes related to motor neuron disease. J Cell Sci 109:2319-2329.

Takeda S, Okabe S, Funakoshi T, Hirokawa N (1994) Differential dynamics of neurofilament-H protein and neurofilament- $L$ protein in neurons. J Cell Biol 127:173-185.

Terada S, Nakata T, Peterson AC, Hirokawa N (1994) Visualization of slow axonal transport in vivo. Science 273:784-788.

Veeranna, Shetty KT, Link WT, Jaffe H, Wang J, Pant HC (1995) Neuronal cyclin-dependent kinase-5 phosphorylation sites in neurofilament protein (NF-H) are dephosphorylated by protein phosphatase 2A. J Neurochem 64:2681-2690.
Wang L, Ho C-L, Sun D, Liem RKH, Brown A (2000) Rapid movement of axonal neurofilaments interrupted by prolonged pauses. Nat Cell Biol 2:137-141.

Watson DF, Griffin JW, Fittro KP, Hoffman PN (1989) Phosphorylation-dependent immunoreactivity of neurofilaments increases during axonal maturation and $\beta, \beta^{\prime}$-iminodipropionitrile intoxication. J Neurochem 53:1818-1829.

Yabe JT, Katchmar T, Shea TB (1997) Initial axonal transport and deposition of neurofilaments is nonlinear and discontinuous. Mol Biol Cell 8[Suppl 1]:280a.

Yabe JT, Pimenta A, Shea TB (1999) Kinesin-mediated transport of neurofilament protein oligomers in growing axons. J Cell Sci 112:3799-3814.

Yabe JT, Chan W, Shea TB (2000) Phospho-dependent association of neurofilament proteins with kinesin in situ. Cell Motil Cytoskeleton 45:249-262. 